\title{
Genotype analysis of noroviruses associated with gastroenteritis outbreaks in childcare centres, Victoria, Australia, 2012-2015
}

\author{
L. D. BRUGGinK, J. M. MOSELEN And J. A. MARSHALL* \\ Victorian Infectious Diseases Reference Laboratory, Doherty Institute, Melbourne, Victoria, Australia
}

Received 24 November 2016; Final revision 5 March 2017; Accepted 15 March 2017;

first published online 11 April 2017

\section{SUMMARY}

The characteristics of norovirus outbreaks in children (0-5 years) in childcare centres in Victoria, Australia (2012-2015) were examined. The three most common open reading frame (ORF) 2 genotypes in childcare centre outbreaks were GII.4 (42\%), GII.6 (21\%) and GII.3 (14\%); the remaining genotypes (GI.2, GI.3, GI.4, GI.8, GI.13, GII.1, GII.2, GII.7 and GII.13) each made up $<10 \%$. The GII.4 genotype was the only norovirus genotype seen in all 4 years of the study and was the most common genotype in 2012-2014 but in 2015 the most common genotype was GII.2. The GII.4 genotype was more common in children 0-2 years, whereas GII.2 and GII.7 were more common in children 4-5 years. ORF 1/ORF 2 recombinant forms identified were GII. P4_NewOrleans_2009/GII.4_Sydney_2012, GII.P12/GII.3, GII.Pb (GII.21)/GII.3, GII.Pe/GII.2, GII.Pe/GII.4_Sydney_2012 and GII.Pg/GII.1. The findings indicate that norovirus genotype prevalence patterns in children were influenced by the age of the children and the year in which the analysis was carried out. The majority of norovirus infections (84\%) occurred after the first year of life so that vaccination before the age of one would appear to be the most efficacious.

Key words: Childcare, genotypes, norovirus, outbreak, vaccine strategies.

\section{INTRODUCTION}

Noroviruses are now considered a major cause of gastroenteritis worldwide [1]. Norovirus outbreaks can occur in virtually any setting where people are in close contact and can affect individuals of all ages [2-4].

Noroviruses are single-stranded positive-sense RNA viruses classified as the genus Norovirus within the family Caliciviridae [3]. The genome consists of three open reading frames (ORFs) that encode a nonstructural polyprotein (ORF 1), the major capsid

\footnotetext{
* Author for correspondence: Dr J. A. Marshall, Victorian Infectious Diseases Reference Laboratory, Doherty Institute, 792 Elizabeth St, Melbourne, Victoria 3000, Australia. (Email: john.marshall@mh.org.au)
}

protein (ORF 2) and a minor structural protein (ORF 3) [3].

Norovirus typing, based on the ORF 2 capsid sequence, has identified six genogroups which are designated GI-GVI [3]. Only genogroups GI, GII and GIV are known to infect humans, with the majority of infections in humans being associated with GII [3]. GIV norovirus infection in humans is poorly understood but appears to be rare [5].

Norovirus genogroups can be further divided into genotypes and these can be based on either ORF 1 or ORF 2 sequencing [6]. As recombination can occur between ORF 1 and ORF 2 regions, a given norovirus can have more than one genotype [7]. The GII.4 genotype is the most common in humans and has been estimated to cause approximately $70-80 \%$ of norovirus-associated gastroenteritis outbreaks 
worldwide [8]. The GII.4 genotype can be further subdivided into 'variants' [6].

Norovirus is an important cause of morbidity and mortality in children in both developed and developing countries. It has been estimated that norovirus accounts for 900000 clinic visits for children per year in developed countries [4] and in the USA, for example, the cost of norovirus treatment in children aged $<5$ years has been estimated at more than US \$273 million annually [9]. In developing countries it has been calculated that norovirus causes up to 200 000 deaths in children $<5$ years old annually [10].

The development of norovirus vaccine strategies requires a detailed knowledge of the nature and incidence of norovirus genotypes in particular age groups but the literature on norovirus in children is incomplete in that most studies on norovirus genotypes in children have focused on children presenting to hospitals rather than those in childcare centres. Although norovirus is well documented in childcare centres [11, 12], norovirus genotype analysis of outbreaks in these centres appears to be rare. Detailed studies on the precise relationship between age of infected individuals and norovirus genotype are also lacking. The current study examines the incidence of norovirus outbreaks in childcare centres in Victoria, Australia over a 4-year period (20122015), and documents the incidence and characteristics of norovirus genotypes associated with these outbreaks.

\section{METHODS}

\section{Outbreak identification}

For the purposes of this study an outbreak of gastroenteritis was defined as an incident, apparently associated with a common event or location, in which four or more individuals had symptoms of gastroenteritis. This study is based on outbreaks for which faecal specimens were sent to the Victorian Infectious Diseases Reference Laboratory (VIDRL) for norovirus testing. VIDRL is the main public health laboratory for viral identification in the state of Victoria, Australia. As such, it receives faecal material from gastroenteritis outbreaks reported to the Victorian Health Department. Only outbreaks that occurred in Victoria were included in the study.

The date of an outbreak was taken as the onset date. If this was not available, the date the outbreak was first notified or the earliest date of collection of a specimen from the outbreak was taken as the date of the outbreak.
Childcare centres were identified on the basis of the description of the facility provided by the Victorian Health Department in the outbreak investigation report. Where faecal specimens were received from a childcare centre outbreak, specimens were only included in the study if they were collected from an individual 5 years old or younger (i.e. all adult carers and older siblings were excluded from the study). For each outbreak, the outbreak age was defined as the average age of all norovirus-positive individuals 5 years old or younger in the outbreak.

\section{Faecal processing and RNA extraction prior to PCR testing}

Faecal specimens were prepared as a $20 \%(\mathrm{v} / \mathrm{v})$ suspension in Hanks' complete balanced salt solution, which was then clarified by centrifugation at $7000 \mathrm{~g}$ for $10 \mathrm{~min}$ [13]. An aliquot of the clarified fluid was then collected and RNA extraction carried out using the Corbett (now Qiagen Sciences, Maryland) automated nucleic acid extraction procedure [14].

\section{RT-PCR, nucleotide sequencing and phylogenetic analysis}

Five two-round RT-PCR protocols were used in the study. Initially all specimens received for norovirus identification were tested by an ORF 1 RT-PCR for GI and GII norovirus. This was carried out with a two-round RT-PCR protocol using primers NV 4562, NV 4611, NV 4692, NV 5296, NV 5298 and NV 5366, as given previously [15]. Nucleotide sequencing analysis of the PCR product derived from this protocol utilised a $440 \mathrm{bp}$ fragment corresponding to nucleotides 4484-4923 (relative to Camberwell virus, AF145896).

Specimens were then tested by an ORF 2 RT-PCR protocol directed at GI noroviruses. This two-round RT-PCR protocol was carried out using primers COG1F and G1SKR as given previously [15]. Nucleotide sequencing analysis of the PCR product derived from the GI protocol utilised a $198 \mathrm{bp}$ fragment corresponding to nucleotides 5415-5612 (relative to Norwalk virus, M87661).

Specimens were also tested by an ORF 2 RT-PCR protocol directed at GII noroviruses. This two-round RT-PCR protocol was carried out using primers G2F3 and G2SKR as given previously [16]. Nucleotide sequencing analysis of the PCR product derived from the GII protocol utilised a $195 \mathrm{bp}$ 
fragment corresponding to nucleotides 5133-5327 (relative to Camberwell virus, AF145896).

Where both ORF 1 and ORF 2 genotypes were available, but gave different designations, a fourth and fifth RT-PCR that cross the ORF 1-ORF 2 intersect were used to try and confirm recombination status in at least one specimen for each genotype combination. These two-round RT-PCR protocols were carried out using primers NV4692 and G2SKR for GIIGII specimens as given previously [15], and primers NV4692 and G1SKR for GII-GI specimens as given previously [16]. Nucleotide sequencing analysis of the PCR product derived from the GII-GII protocol utilised a fragment in the range 345-753 bp corresponding to nucleotides 4484-5273 (relative to Camberwell virus, AF145896). The GII-GI protocol did not yield any product.

Nucleotide sequencing and phylogenetic analysis were carried out using the software MacVector software (Oxford Molecular Limited, Madison, WI, USA), Phylip [17] and FigTree (available at http:// tree.bio.ed.ac.uk/software/figtree/). Genotype analysis also made use of the norovirus-automated genotyping tool (http://www.rivm.nl/mpf/norovirus/typing tool) [18].

\section{Experimental plan}

Between 1 and 6 specimens were received from children in each childcare centre outbreak.

Every such specimen (other than duplicate specimens from the same individual in a given outbreak) was then processed and tested by the ORF $1 \mathrm{RT}-$ PCR protocol, the ORF 2 GI RT-PCR protocol and the ORF 2 GII RT-PCR protocol. An outbreak was designated as norovirus positive if at least one norovirus positive specimen was identified by one of these protocols in a given outbreak.

An attempt was made to sequence every norovirus RT-PCR-positive specimen (by all three RT-PCR protocols above). In addition, where both an ORF 1 and ORF 2 genotype was available, but gave different genotype designations, further RT-PCR protocols were carried out to try to confirm the recombination status of these specimens.

\section{Statistical analysis}

Statistical analysis made use of three procedures: the $\chi^{2}$ test, the partitioning of $\chi^{2}$ procedure [19] and Fisher's exact two-tailed test [20].

\section{RESULTS}

\section{Incidence and seasonal periodicity}

In the 4-year period 2012-2015, 1076 gastroenteritis outbreaks were received and $697(64.8 \%)$ were found to be norovirus positive. Out of the 1076 gastroenteritis outbreaks, 91 outbreaks were received from childcare centres. Of these 37 were positive by the ORF 1 RT-PCR and a further 11 were negative by the ORF 1 RT-PCR but positive by one of the ORF 2 RT-PCRs. Thus a total of $48(52.7 \%)$ of all outbreaks received from childcare centres were positive for norovirus, indicating it was an important cause of viral gastroenteritis outbreaks in childcare centres.

The cumulative periodicity of norovirus-positive outbreaks over the 4-year period is shown in Fig. 1. A difference in the seasonality of outbreaks in childcare centre and non-childcare centre outbreaks was found in that $40 \%$ of the outbreaks in childcare centre outbreaks occurred in the period February-June compared with only $23 \%$ for non-childcare centre outbreaks; the difference was statistically significant $\left(P<0.025, \chi^{2}\right.$ test $)$.

\section{Genotypes detected in children}

A broad range of norovirus genotypes were detected over the 4-year period of the study (Table 1). ORF 2 genotypes were characterised in 43 outbreaks and of these the three most common genotypes (i.e. $>10 \%$ ) were GII.4 (42\%), GII.6 (21\%) and GII.3 (14\%); all of the remaining ORF 2 genotypes (GI.2, GI.3, GI.4, GI.8, GI.13, GII.1, GII.2, GII.7 and GII.13) each made up $<10 \%$ of the total (Table 1). Phylogenetic trees showing the relationships between all unique ORF 2 sequences (except GI.13) are given in Fig. 2.

From Table 1 it can also be seen that there were a number of potential ORF 1/ORF 2 recombinant forms (i.e. noroviruses where the genotypes or genotype variants in ORF 1 and ORF 2 were different). Application of the ORF 1-ORF 2 RT-PCRs confirmed the identity of six recombinant forms. These recombinants and their associated GenBank numbers were as follows: GII.P4_NewOrleans_2009/ GII.4_Sydney_2012 (KX789172), GII.P12/GII.3 (KX064757), GII.Pb(GII.21)/GII.3 (KX064758), GII. Pe/GII.2 (KX064759), GII.Pe/GII.4_Sydney_2012 (KX789173) and GII.Pg/GII.1 (KR338971) [21]. 


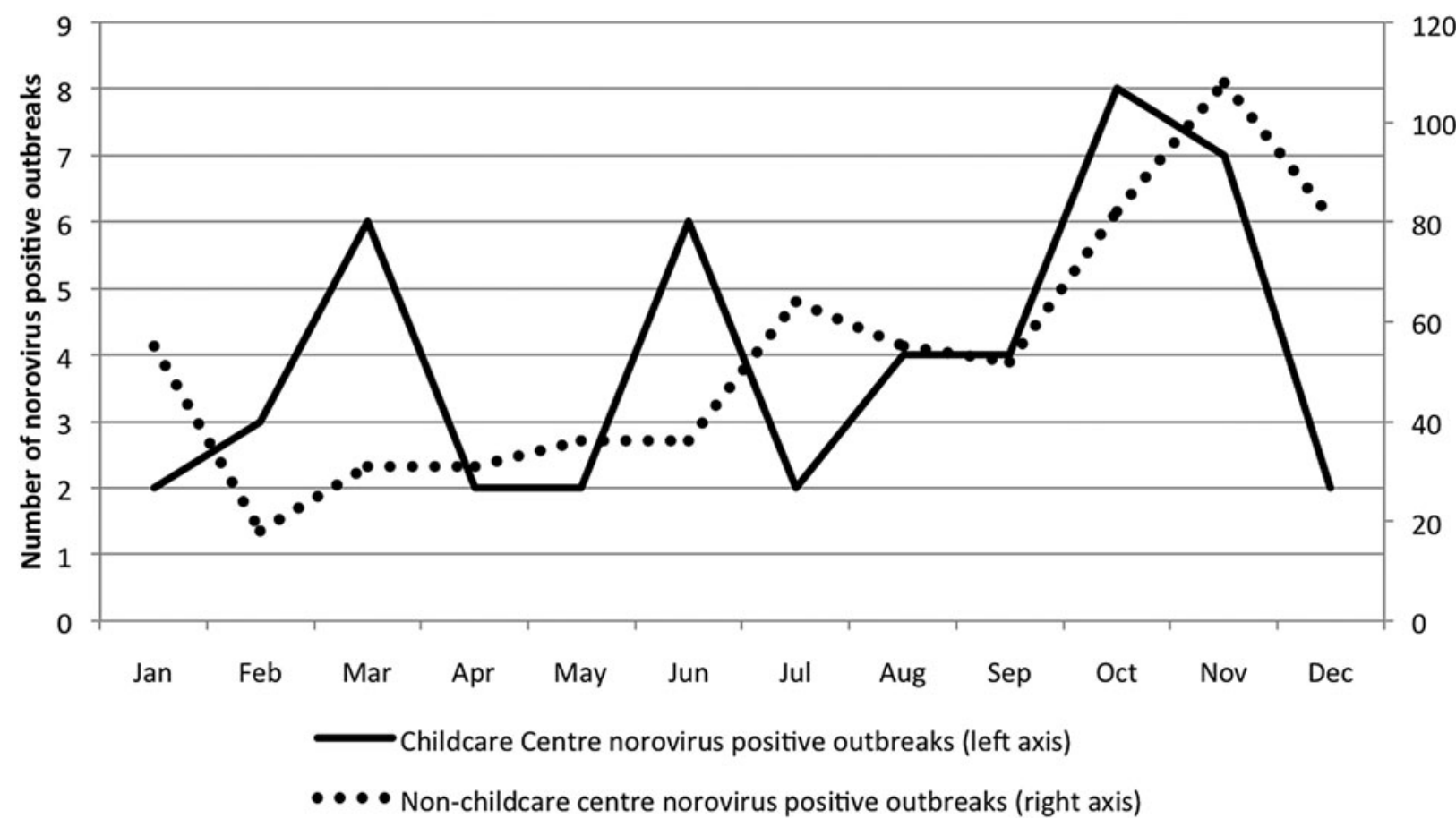

Fig. 1. The 4-year cumulative periodicity of norovirus-positive outbreaks from childcare centres (left axis) compared with norovirus-positive outbreaks from all other settings (i.e. non-childcare centre outbreaks) (right axis).

Table 1. Norovirus ORF 1 and ORF 2 genotype combinations observed in childcare centre outbreaks, 2012-2015

\begin{tabular}{lll}
\hline \hline ORF 1 genotype & ORF 2 genotype & Number of norovirus-positive outbreaks \\
\hline GI.P2 & GI.2 & 1 \\
GI.P2 or no sequence available* & GI.2 or GII.6* & 1 \\
GI.P8 & GI.8 & 1 \\
GII.P4_DenHaag_2006b & GII.4† & 1 \\
GII.P4_NewOrleans_2009 & GII.4_Sydney_2012 & 1 \\
GII.P12 & GII.3 & 3 \\
GII.P12 & GI.3 \& GII.3* & 1 \\
GII.P12 & no sequence available & 1 \\
GII.P16 & GII.13 & 1 \\
GII.Pb (GII.P21) & GII.3 & 2 \\
GII.Pb (GII.P21) & GII.6 & 1 \\
GII.Pb or GII.Pe* & GII.4_Sydney_2012 & 1 \\
GII.Pe & GII.2 & 1 \\
GII.Pe & GII.4_Sydney_2012 & 6 \\
GII.Pe & GII.4† & 8 \\
GII.Pe & No sequence available & 4 \\
GII.Pg & GII.1 & 2 \\
No sequence available & GI.13 \& GII.4_Sydney_2012ł or GI.4* & 1 \\
No sequence available & GII.2 & 2 \\
No sequence available & GII.2 or GII.6* & 1 \\
No sequence available & GII.6 & 5 \\
No sequence available & GII.6 or GII.7* & 1 \\
No sequence available & GII.7 & 2 \\
Total & & 48 \\
\hline \hline
\end{tabular}

* Mixed outbreak with different individuals giving different genotypes.

$\dagger$ GII.4 variant could not be determined.

$\$$ Mixed infection of two genotypes in a single individual. 


\section{Variation in ORF 2 genotype incidence, 2012-2015}

The changing temporal pattern of ORF 2 norovirus genotypes found in childcare centre outbreaks is given in Table 2. It can be seen that GII.4 norovirus was present in all 4 years of the study, GII.3 and GII.6 in three of the 4 years, GI.2 and GII.7 in 2 years and all the remaining genotypes (GI.3, GI.4, GI.8, GI.13, GII.1, GII.2, and GII.13) in only 1 year of the study.

The relative importance of the GII.4 genotype varied from year to year. In 2 years of the study (2012 and 2014) GII.4 made up over $50 \%$ of outbreaks of known genotype. In 2013 GII.4 was the most common genotype but made up $<50 \%$ of outbreaks of known genotype. However, in 2015 GII.4 was only found in 23\% of outbreaks of known genotype and occurred at the same frequency as GII.3 and GII.6 in that year; the most common genotype in 2015 was GII.2.

\section{The relationship between age, norovirus outbreak incidence and norovirus genotype}

The relationship between age and norovirus incidence was first examined for the outbreaks of known ORF 2 genotype and it was found that the outbreak age of most of the outbreaks $(84 \%)$ was $>1$ year (Table 3 ). In particular, norovirus incidence tended to occur more commonly in the 1 to $<2$ year age group, with statistical analysis confirming that the number of outbreaks in the 1 to $<2$ year age group was significantly higher $\left(P<0.001\right.$, partitioning of $\left.\chi^{2}\right)$ than in any other age group (Table 3 ).

A broad range of norovirus genotypes were detected in the study (Table 3). Although for most genotypes there was no simple relationship between incidence and age, for three genotypes (GII.4, GII.2 and GII.7) there was. In particular, GII.4 norovirus was significantly more common in outbreaks involving children aged 0 2 years than in outbreaks involving children aged 3-5 years $\left(P<0.05, \chi^{2}\right.$ test). Thus, GII.4 norovirus tended to occur more commonly in younger children. In contrast, GII.2 norovirus was significantly more common in outbreaks involving children aged 4-5 years than in outbreaks involving children aged $0-4$ years $(P<$ 0.0033, Fisher's exact two-tailed test). Similarly, GII.7 norovirus was significantly more common in outbreaks involving children aged 4-5 years than in outbreaks involving children aged $0-4$ years $(P<0.0033$, Fisher's exact two-tailed test). Thus, GII.2 and GII.7 norovirus tended to occur more commonly in older children.

\section{DISCUSSION}

The current consensus is that one of the primary target groups for norovirus vaccination should be young children, as this group has one of the greatest disease burdens [22-26]. Vaccine strategies, however, require a thorough knowledge of what genotypes are found in children and how or whether they change over time. This was the subject of the current study.

A review of recent studies of norovirus genotypes in children revealed that studies of this type were common. However, community-based outbreaks were not considered and in all studies identified in the literature only hospital cases were analysed. The problem with using hospital cases alone and considering each individual to be separate and distinct from other cases is that there is a risk that the data will give a slanted view of norovirus genotype prevalence, as hospital-based data may be sampling multiple individuals from the same cluster. The current study attempted to give a broader view of the topic by using community-based gastroenteritis outbreaks as the data source and by focusing on little discussed topics, such as how norovirus genotypes in children vary over time and the relationship between norovirus genotype and the age of the infected child.

Norovirus outbreaks in the current study showed a distinctive seasonal pattern, with a non-epidemic period in the early part of the year and then an epidemic peak in the latter part of the year. However, a difference in the seasonality of outbreaks in childcare centres compared with all other outbreaks was found, in that the seasonality of outbreaks in childcare centres was less pronounced than the seasonality of outbreaks in other settings. Thus seasonal factors, while important, were less influential in childcare norovirus outbreaks than in all other norovirus outbreaks.

A broad range of norovirus genotypes were detected over the 4 years of the study and the principal genotypes found were the same as those found in a hospital-based study of children in the same age group over a similar time period [27]. The three most common ORF 2 genotypes in the current study were GII.4 (42\%), GII.6 (21\%) and GII.3 (14\%). In this regard the findings accord with those of Chan et al. [27], who examined norovirus ORF 2 genotypes in hospitalised children ( $\leqslant 5$ years) in Hong Kong in the period 2012-2014 and found that GII.4 was by far the most common, followed by GII.6 and then GII.3.

It is also worth noting that the findings of the current study accord closely with those of Brown et al. 
(a)

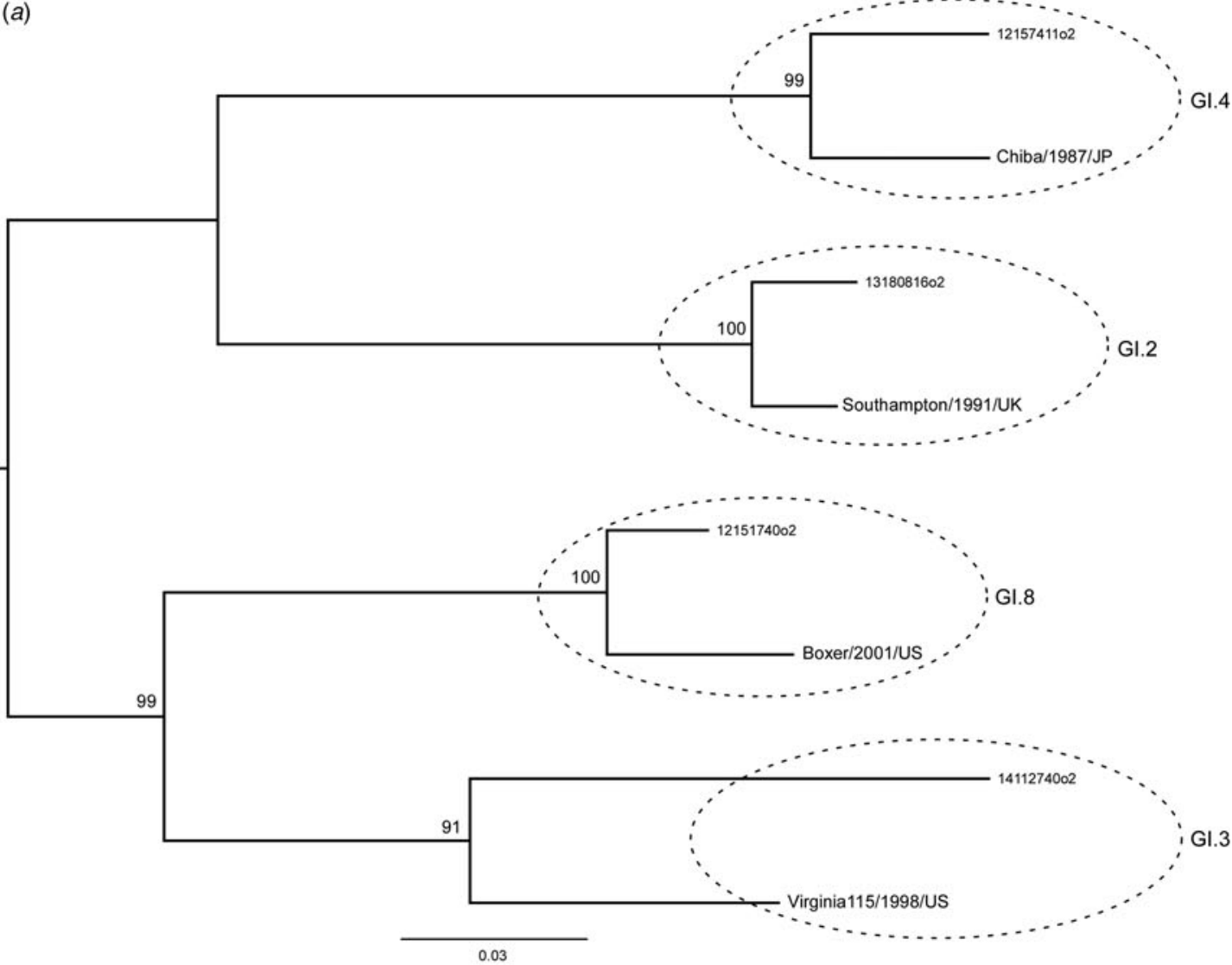

(b)

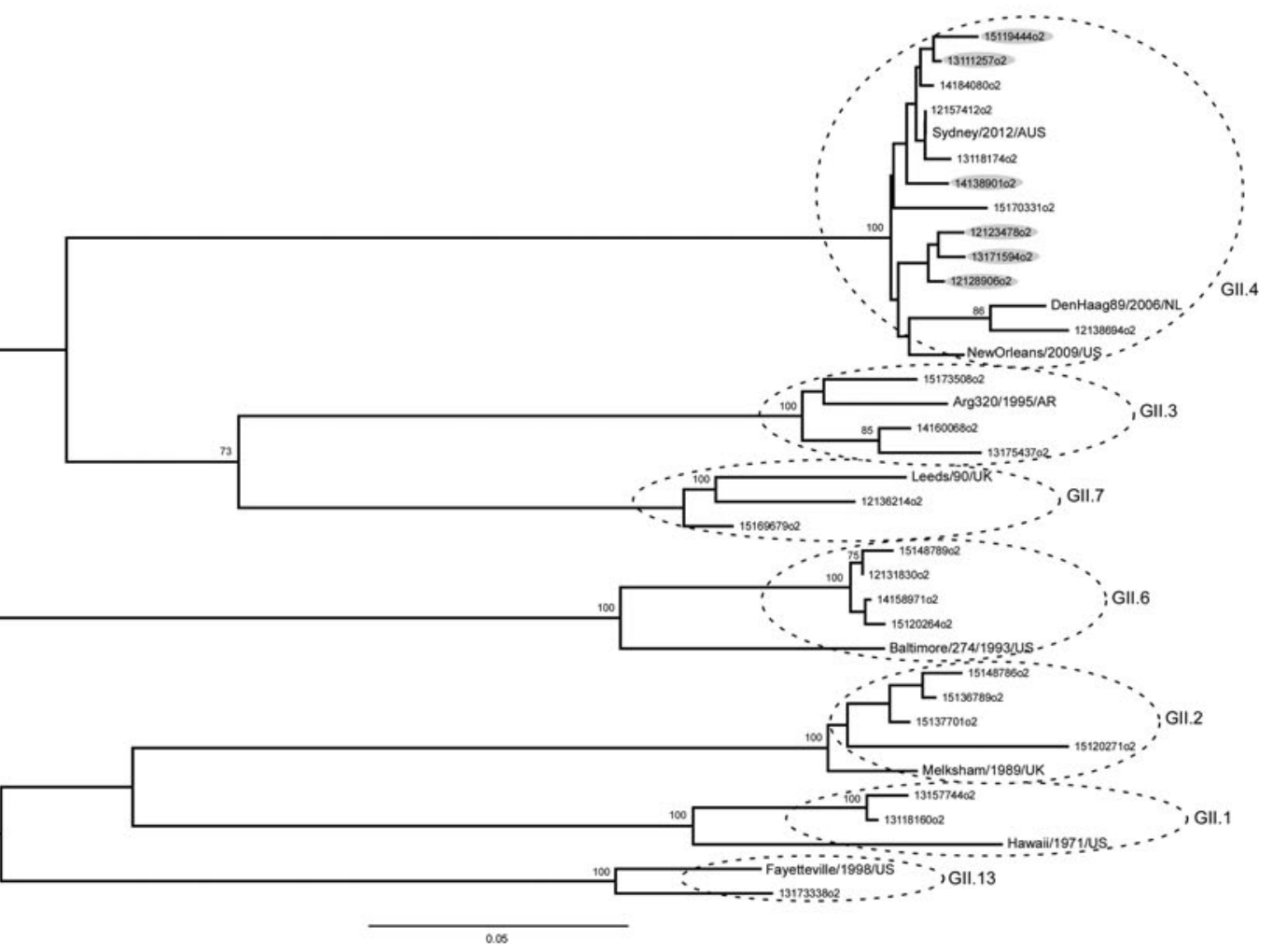

Fig. 2. DNA (dist) Kimura phylogenetic trees showing the relationship of ORF 2 GI (Fig. 2a) and GII (Fig. 2b) genotypes detected. The GI tree was based on an ORF 2 sequence 198 bp long and the GII tree was based on an ORF 2 
Table 2. Norovirus ORF 2 genotypes detected by year (2012-2015)

\begin{tabular}{lllll}
\hline \hline & \multicolumn{4}{l}{ Number of outbreaks } \\
\cline { 2 - 5 } ORF 2 genotype & 2012 & 2013 & 2014 & 2015 \\
\hline GI.2 & 0 & 1 & 0 & 0 \\
GI.2 or GII.6* & 0 & 0 & 1 & 0 \\
GI.3 \& GII.3† & 0 & 0 & 1 & 0 \\
GI.8 & 1 & 0 & 0 & 0 \\
GI.13 \& GII.4† or GI.4* & 1 & 0 & 0 & 0 \\
GII.1 & 0 & 2 & 0 & 0 \\
GII.2 & 0 & 0 & 0 & 3 \\
GII.2 or GII.6* & 0 & 0 & 0 & 1 \\
GII.3 & 0 & 1 & 1 & 3 \\
GII.4 & 6 & 3 & 5 & 3 \\
GII.6 & 3 & 0 & 1 & 2 \\
GII.6 or GII.7* & 1 & 0 & 0 & 0 \\
GII.7 & 1 & 0 & 0 & 1 \\
GII.13 & 0 & 1 & 0 & 0 \\
No sequence available & 2 & 0 & 2 & 1 \\
Total & 15 & 8 & 11 & 14 \\
\hline \hline
\end{tabular}

* Mixed outbreak with different individuals giving different genotypes.

$\dagger$ Mixed infection of two genotypes in a single individual.

[28] who examined prevalence patterns of norovirus genotypes in paediatric inpatients in a British hospital in the period 2014-2015. Depending on their clinical classification the patients had a median age varying from 1.5 to 3.3 years although individuals as old as 18 were also included in the study. These authors found that the four most common norovirus genotypes detected were GII.4 (52\%), GII.3 (26\%), GI.3 $(9 \%)$ and GII.6 (5\%). The high detection rate of GI.3 in the study of Brown et al. [28] compared with the current study may reflect the broader age range sampled in the study of Brown et al. [28].

However, care is needed in making broad generalisations on norovirus prevalence patterns in children, as the results of the current study indicate temporal factors are also important. Thus it was notable that
GII.4 was only found in the majority of outbreaks in 2 years (2012 and 2014) although it was the most common genotype in 3 years of the study (2012, 2013 and 2014). In 2015 GII.4 was only found in $23 \%$ of norovirus outbreaks and occurred at the same frequency as GII.3 and GII.6 in that year; the most common genotype was GII.2. Thus the incidence of norovirus genotypes in childcare centres can vary markedly from year to year.

In the current study norovirus outbreak incidence was found to be linked to the age of the children. Thus outbreaks among children aged 1 to $<2$ years were significantly more common than in children aged $<1$ year and children aged $2-5$ years, so that norovirus susceptibility peaked in the second year of life in young children.

It was also noted there was a significant link between age and the incidence of some norovirus genotypes. Thus GII.4 norovirus was more common in very young children ( $0-2$ years) than in older children (3-5 years). On the other hand, GII.2 and GII.7 norovirus were significantly more common in older children ( $4-5$ years) than in younger children ( $0-4$ years).

The results of the current study also give some indication of the age at which children should be vaccinated against norovirus. It was found that most infections in children (84\%) occurred from the age of one, so early vaccination would appear to be the most efficacious. This is in agreement with the study of Shioda et al. [29], who proposed that norovirus immunisation should be completed by 6 months as this would prevent approximately $85 \%$ of cases.

The findings of the current study indicate that norovirus genotype prevalence patterns in children can be complex and are influenced by two sets of variables, the age of the infected child and the calendar year in which the study was carried out. The study also provides independent data that early norovirus vaccination of children would appear to be the most effective.

sequence $195 \mathrm{bp}$ long. Only unique sequences were included in the trees. The reference strains included in the trees were as follows: Arg320/1995/AR (AF190817), Baltimore/274/1993/US (AF414408), Boxer/2001/US (AF538679), Chiba/1987/JP (AB042808), DenHaag89/2006/NL (EF126965), Fayetteville/1998/US (AY113106), Hawaii/1971/US (U07611), Leeds/90/ UK (AJ277608), Melksham/1989/UK (X81879), NewOrleans/2009/US (GU445325), Southampton/1991/UK (L07418), Sydney/2012/AUS (JX459908) and Virginia115/1998/US (AY038598). In Fig. 2b all GII.4 noroviruses that could not be assigned to a variant form are shaded. The genotype GI.13 could not be incorporated in the GI tree as the sequence had an extra codon and therefore could not be made to match the sequence length of the other GI genotypes. The figures on the branches represent bootstrap values (\%) after resampling 1000 datasets. Only bootstrap values $\geqslant 70 \%$ are shown. The scale marker represents substitutions per site. 
Table 3. Outbreak ages* compared with ORF 2 genotypes detected

\begin{tabular}{|c|c|c|c|c|c|c|}
\hline \multirow[b]{2}{*}{ ORF 2 genotypes found in outbreaks } & \multicolumn{6}{|c|}{ Outbreak age* } \\
\hline & $<1$ year & 1 to $<2$ years & 2 to $<3$ years & 3 to $<4$ years & $4-5$ years & Total \\
\hline GI.2 & & & & 1 & & 1 \\
\hline GI.2 or GII.6† & & 1 & & & & 1 \\
\hline GI.3 \& GII.3\$ & 1 & & & & & 1 \\
\hline GI.8 & & & & 1 & & 1 \\
\hline GI.13 \& GII. $4 \ddagger$ or GI. $4 \dagger$ & & 1 & & & & 1 \\
\hline GII.1 & 1 & 1 & & & & 2 \\
\hline GII.2 & & 1 & & & 2 & 3 \\
\hline GII.2 or GII. $6 \dagger$ & & & 1 & & & 1 \\
\hline GII.3 & 1 & 2 & & 2 & & 5 \\
\hline GII.4 & 4 & 10 & 1 & 2 & & 17 \\
\hline GII.6 & & 3 & 3 & & & 6 \\
\hline GII.6 or GII. $7 \dagger$ & & & 1 & & & 1 \\
\hline GII.7 & & & & & 2 & 2 \\
\hline GII.13 & & 1 & & & & 1 \\
\hline Total & 7 & 20 & 6 & 6 & 4 & 43 \\
\hline
\end{tabular}

* Outbreak ages were calculated by averaging the ages of all norovirus-positive individuals in a given outbreak.

$\dagger$ Mixed outbreak with different individuals giving different genotypes.

$\$$ Mixed infection with one individual having multiple genotypes.

\section{REFERENCES}

1. Thorne LG, Goodfellow IG. Norovirus gene expression and replication. Journal of General Virology 2014; 95 : 278-291.

2. Bruggink L, Marshall J. The relationship between health care and nonhealth care norovirus outbreak settings and norovirus genotype in Victoria, Australia, 2002-2005. Journal of Microbiology, Immunology and Infection 2011; 44: 241-246.

3. Green KY. Caliciviridae: the Noroviruses. In: Knipe DM, Howley PM, eds. Fields Virology, 6th edn, Volume 1. Philadelphia: Wolters Kluwer/Lippincott Williams \& Wilkins, 2013, pp. 582-608.

4. Robilotti E, Deresinski S, Pinsky BA. Norovirus. Clinical Microbiology Reviews 2015; 28: 134-164.

5. La Rosa G, et al. Detection of genogroup IV noroviruses in environmental and clinical samples and partial sequencing through rapid amplification of cDNA ends. Archives of Virology 2008; 153: 2077-2083.

6. Kroneman A, et al. Proposal for a unified norovirus nomenclature and genotyping. Archives of Virology 2013; 158: 2059-2068.

7. Bull RA, Tanaka MM, White PA. Norovirus recombination. Journal of General Virology 2007; 88: 3347-3359.

8. de Graaf M, et al. Emergence of a novel GII.17 norovirus - End of the GII.4 era? Euro Surveillance 2015; 20(26): $\mathrm{p}$ ii $=21178$.

9. Payne DC, et al. Norovirus and medically attended gastroenteritis in U.S. children. The New England Journal of Medicine 2013; 368: 1121-1130.

10. Patel MM, et al. Systematic literature review of role of noroviruses in sporadic gastroenteritis. Emerging Infectious Diseases 2008; 14: 1224-1231.
11. Enserink R, et al. Gastroenteritis attributable to 16 enteropathogens in children attending day care. Significant effects of rotavirus, norovirus, astrovirus, Cryptosporidium and Giardia. The Pediatric Infectious Disease Journal 2015; 34: 5-10.

12. Pijnacker R, et al. Characteristics of child daycare centres associated with clustering of major enteropathogens. Epidemiology and Infection 2016; 144: 2527-2539.

13. Witlox KJ, et al. The effect of fecal turbidity on norovirus detection by reverse transcriptase polymerase chain reaction. Diagnostic Microbiology and Infectious Disease 2010; 66: 230-232.

14. Witlox KJ, et al. A comparative evaluation of the sensitivity of two automated and two manual nucleic acid extraction methods for the detection of norovirus by RT-PCR. Journal of Virological Methods 2008; 150: 70-72.

15. Bruggink LD, et al. Molecular and epidemiological features of gastroenteritis outbreaks involving genogroup I norovirus in Victoria, Australia, 2002-2010. Journal of Medical Virology 2012; 84: 1437-1448.

16. Bruggink LD, Dunbar NL, Marshall JA. Norovirus genotype diversity in community-based sporadic gastroenteritis incidents: a five-year study. Journal of Medical Virology 2015; 87: 961-969.

17. Felsenstein J. PHYLIP - Phylogeny Inference Package (Version 3.2). Cladistics 1989; 5: 164-166.

18. Kroneman A, et al. An automated genotyping tool for enteroviruses and noroviruses. Journal of Clinical Virology 2011; 51: 121-125.

19. Agresti A. Categorical Data Analysis. New York: John Wiley and Sons, Inc., 1990, pp. 50-53. 
20. Hays WL. Statistics for Psychologists. New York: Holt, Rinehart and Winston, 1963, pp. 598-601.

21. Bruggink LD, Dunbar NL, Marshall JA. Emergence of GII.Pg norovirus in gastroenteritis outbreaks in Victoria, Australia. Journal of Medical Virology 2016; 88: $1521-1528$.

22. Aliabadi $\mathbf{N}$, et al. Progress toward norovirus vaccines: considerations for further development and implementation in potential target populations. Expert Review of Vaccines 2015; 14: 1241-1253.

23. Bartsch SM, et al. The potential economic value of a human norovirus vaccine for the United States. Vaccine 2012; 30: 7097-7104.

24. Debbink K, Lindesmith LC, Baric RS. The state of norovirus vaccines. Clinical Infectious Diseases 2014; 58: 1746-1752.

25. DiCaprio E, et al. Epidemiology, prevention, and control of the number one foodborne illness: human norovirus. Infectious Disease Clinics of North America 2013; 27: 651-674.

26. Lindesmith LC, et al. Broad blockade antibody responses in human volunteers after immunization with a multivalent norovirus VLP candidate vaccine: immunological analyses from a phase I clinical trial. PLoS Medicine 2015; 12: e1001807.

27. Chan MCW, et al. Virus genotype distribution and virus burden in children and adults hospitalized for norovirus gastroenteritis, 2012-2014, Hong Kong. Scientific Reports 2015; 5: 11507.

28. Brown JR, Shah D, Breuer J. Viral gastrointestinal infections and norovirus genotypes in a paediatric UK hospital, 2014-2015. Journal of Clinical Virology 2016; 84: $1-6$.

29. Shioda K, et al. Global age distribution of pediatric norovirus cases. Vaccine 2015; 33: 4065-4068. 